\title{
MODELING THE CONTROL PROCESSES OF THE SIZE POPULATION OF MICROORGANISMS USING MODULATED MICROWAVE
}

\author{
Alexander Oshchepkov \\ Perm State University \\ Russia \\ Aos57@mail.ru
}

Article history:

Received 03.09.2021, Accepted 25.11.2021

\begin{abstract}
The paper describes a mathematical model of a control system for the size population of microorganisms using modulated microwave radiation of non-thermal power. The model is implemented in the dynamic environment MATLAB+Simulink, the areas of optimal values of the impact parameters are found. An algorithm for automatic tuning of the modulation frequency during the experiment has been developed. The efficiency of the algorithm is shown with the help of computer simulation.
\end{abstract}

\section{Key words}

Microwave, control system, optimization, resonant absorption.

\section{Introduction}

The tasks of controlling the size of the population of unicellular microorganisms using external influences consist either in increasing the population (for example, accelerating the growth of beneficial microorganisms), or in decreasing the population up to complete destruction (combating pathogenic bacteria, tumor cells). This paper considers the use of microwave radiation of non-thermal power to solve the second problem. The inhibitory effect on unicellular organisms of an alternating electromagnetic field of low power, harmless to humans, was discovered experimentally [Kozmin, 2006]. In the research article [Ikhlov, 2020], the resonant nature of this effect is noted, due to the absorption of radiation energy by DNA molecules of cells at certain frequencies equal to the natural frequencies of torsional vibrations of molecules. Due to the large size of DNA macromolecules, the absorption mechanism can be described classically. The presence of frequencies in the microwave range in the spectrum of torsional vibrations of the DNA molecule was established by calculation within the framework of the coarse-grained model of the DNA double helix [Kovaleva, 2009].

The effectiveness of the action of an alternating field of a given frequency is proportional to the magnitude of absorption at a given frequency. From the experimental data it follows that the absorption line is very narrow (the quality factor is 100 or more), therefore, the deviation of the exposure frequency from the resonant frequency leads to a sharp decrease in the effectiveness of the exposure. In this paper, to improve efficiency under conditions of uncertainty of the resonant frequency, it is proposed to use modulation of the fundamental frequency of the action at a constant radiation power. The modulation frequency and modulation index [Baskakov, (2016)] are the parameters that determine the effectiveness of the impact. To automatically determine the most effective values of these parameters, an optimization algorithm has been developed using the feedback principle. The operation of the control system with the optimization block was demonstrated on a computer model in the MATLAB + Simulink environment. The advantages of using optimized modulated radiation under conditions of uncertainty of the required frequency of exposure are estimated.

\section{Mathematical model of the control system}

The object of management is the size of the population of microorganisms. Microwave radiation plays the role of a control action. The goal of control is to reduce the population to zero. Let's compose a mathematical model of the control system. 
In the case of small oscillations, the alternating electric field of the radiation wave deflects the average dipole moment of the DNA molecule from the equilibrium position. In the presence of dissipation and a restoring moment of forces due to the interaction of the molecule with the environment, forced torsional vibrations arise. Within the framework of the classical approach, the dynamics of the angle of rotation $\varphi$ can be described by the equation of oscillations of an oscillator with friction:

$$
\ddot{\varphi}+\lambda \dot{\varphi}+\omega_{0}^{2} \varphi=A \cos (\Phi(t)),
$$

where $\omega_{0}$ is the resonant frequency of the oscillator, $\lambda=2 \omega_{0} / Q$ is the coefficient of friction in frequency units, $Q$ is the quality factor. The constant value $\mathrm{A}$ is proportional to the amplitude of the electric field of the wave, the total phase $\Phi(t)$ with single-tone modulation [Baskakov, (2016)] has the form:

$$
\Phi(t)=\omega_{C} t+\frac{\Delta F}{F_{m}} \sin \left(2 \pi F_{m} t\right),
$$

where $\omega_{C}$ is the center frequency, $F_{m}$ is the modulation frequency, $\Delta F$ is the frequency deviation.

Ratio

$$
m=\frac{\Delta F}{F_{m}}
$$

called the modulation index.

The energy absorption is proportional to the mean square of the angle of rotation over a sufficiently long time interval $\mathrm{T}$ :

$$
I=\frac{1}{T} \int_{t_{0}}^{T} \varphi^{2}(t) d t
$$

$t_{0}$ - onset of radiation action. The absorption $I$ is a function of the radiation parameters: $I=I\left(\omega_{c}, F_{m}, \Delta F\right)$.

Substitution of a particular solution of equation (2.1) for $\Delta F=0$ [Landau, 1988] into (4) after calculating the integral leads to the expression for $I$ : $I=\frac{A^{2}}{2 \omega_{0}{ }^{4}} \cdot W(\gamma)$, where the dimensionless absorption function $W$ has the form:

$$
W(\gamma)=\frac{1}{\left(1-\gamma^{2}\right)^{2}+4 \gamma^{2} / Q^{2}}, \gamma=\frac{\omega_{C}}{\omega_{0}} .
$$

The maximum of function (5) is attained at $\gamma_{\text {res }}=\sqrt{1-2 / Q^{2}}$ (resonance condition). Thus, the deviation of the resonant frequency from the value $\omega_{0}$ due to dissipation at is less than $0,01 \%$. We will further consider the cases when the uncertainty of the resonance frequency is several percent, the absorption of radiation drops sharply, which leads to the need for modulation.

Relations (1) - (4) represent a mathematical model of the microscopic interaction of modulated radiation with microorganisms. To study the effect of radiation on the population as a whole, it is necessary to draw up its dynamic model.

In [Oshchepkov, 2018], a phenomenological model of population dynamics is proposed, which is a modification of the Verhulst equation [Fursova, 2008] in the presence of external influences:

$$
\dot{N}=\frac{1}{\tau}\left(p_{1}(t)-p_{2}(t) \frac{N}{N_{C}}\right) \cdot N,
$$

where $N$ is the number of microorganisms in the population, $\tau=\Delta T / \ln 2, \Delta T$ is the average time of cell division (generation period), $N c$ is the capacity of the medium, parameters $p_{1}>0, p_{2}>0$ describe the external effect.

It is assumed before the start of exposure $p_{1}=1, \quad p_{2}=1$ (Verhulst model). Parameter $p_{1}$ takes into account the phenomenon of hormesis [Kuzin, (1991)]: the exposure inhibits cell division, and at the first stage their number increases. Parameter $p_{2}$ describes the effectiveness of the inhibitory effect of radiation on the population, which consists in an exponential decrease in the capacity of the medium from $N c$ to a certain minimum value of $N_{\infty}$. The time dependence of the parameters is as follows:

$$
\begin{aligned}
& p_{1}(t)=1+\alpha(t), \\
& \alpha(t)= \begin{cases}0, & t<0 ; \\
\alpha_{0} e^{-t / \tau_{\alpha}}, & t \geq 0 .\end{cases} \\
& p_{2}(t)=(1-\beta(t))^{-1}, \\
& \beta(t)= \begin{cases}0, & t<0 ; \\
\beta_{\infty}\left(1-e^{-t / \tau_{\beta}}\right), & t \geq 0 ;\end{cases} \\
& \beta_{\infty}=\frac{N_{C}-N_{\infty} .}{N_{C}}
\end{aligned}
$$

Parameter $\beta_{\infty}$ is a measure of the effectiveness of the impact: at $\beta_{\infty}=0$, radiation has no effect on the population, at $\beta_{\infty}=1$, radiation completely destroys microorganisms $\left(N_{\infty}=0\right)$. Obviously, $\beta_{\infty}$ is proportional to the absorption (4):

$$
\beta_{\infty}=I / I_{\max }, \quad I_{\max }=I\left(\omega_{c}=\omega_{0}, \Delta F=0\right) .
$$

Hence, the goal of control can be formulated as

$$
\beta_{\infty} \rightarrow 1 \text {. }
$$

The mathematical model of the control system is built.

\section{Computer modeling of the process of exposure to radiation on an object}

We will consider radiation in the centimeter range, the frequency of which is $10^{9} \ldots 10^{10} \mathrm{~Hz}$, respectively, the oscillation period lies in the range of $10^{-10} \ldots 10^{-9} \mathrm{~s}$. The division time of unicellular organisms ranges from several tens of minutes to several hours, i.e. from $10^{3}$ to $10^{4} \mathrm{~s}$. Consequently, the characteristic times in 
equations (1) and (6) differ by 12 or more orders of magnitude. Therefore, the simulation is carried out in stages: first, equation (1) is solved and the dependence of absorption $\mathrm{W}$ on the radiation parameters is determined, then the obtained dependence through relation (8) is used to modelling the dynamic equation (6), which simulates a real experiment. This approach allows one to study optimization algorithms that are experimentally feasible.

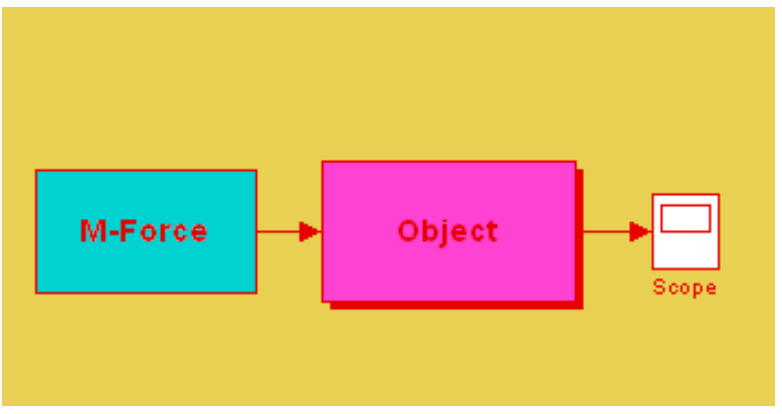

Figure 1. S-model for the study of the absorption process

Fig. 1 shows the S-model for studying the absorption process. The "M-Force" block forms the modulated signal on the right side of equation (1), the "Object" block solves equation (1). The output signal is displayed on a virtual oscilloscope and transferred to the external program "Modul_f", from which the Smodel is started.

The program calculates the dimensionless absorption function $W$ in accordance with equation (4) and plots the necessary graphs. Simulation time is measured in frequency characteristics: $F_{0}=1$, the decrement $\lambda=0,01$, simulation time $T=200$, the simulation step is 0,05 , and convenient dimensionless parameters are introduced:

$$
d=\frac{\Delta F}{F_{c}}, \quad b=\frac{F_{m}}{F_{c}}, \quad D F=\frac{F_{c}-F_{0}}{F_{0}} .
$$

Parameter $d$ is the dimensionless frequency deviation, $b$ is the dimensionless modulation frequency, $D F$ is the deviation of the radiation frequency from the resonant one. Parameters $b$ and $D F$ below can be given in percentages. The modulation index (3) is then determined by the expression

$$
m=\frac{d}{b} \text {. }
$$

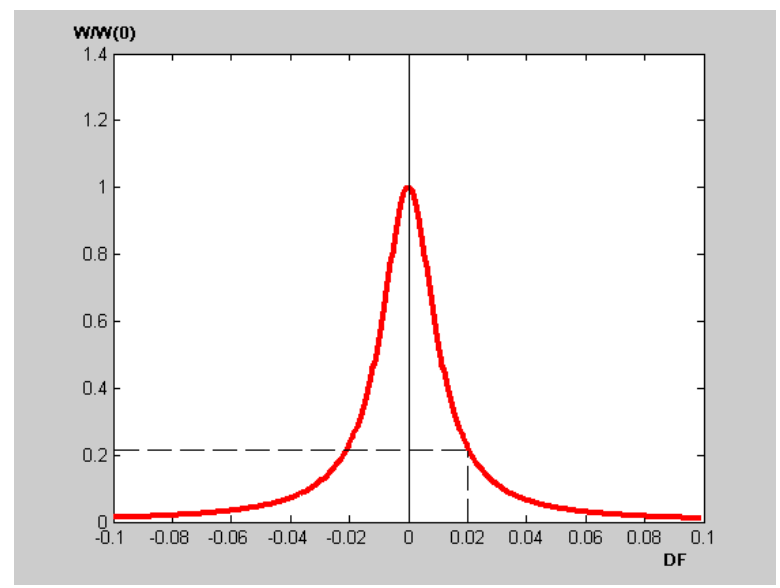

Figure 2. Absorption curve in the absence of modulation

The graph of the dependence of the absorption function $W$ on $D F$ at $d=0$ is shown in Fig. 2. In this case, the program reproduces the dependence determined by formula (5). We see that for the selected parameters of the object, the deviation of the frequency from the resonant frequency, for example, by $2 \%$, reduces the absorption by a factor of 5 : $W(D F=0,02) \approx 0,2$.

The graphs in Fig. 3, show the role of modulation of the radiation frequency. They show the dependences of the absorption function $W b$ for $D F=+0,02$ and $D F=-0,02$ on the dimensionless modulation frequency $b$ at a fixed modulation index $m=2$. Note that, in accordance with definition (8), the values of the function $W b$ coincide with the values of the parameter $\beta_{\infty}$.

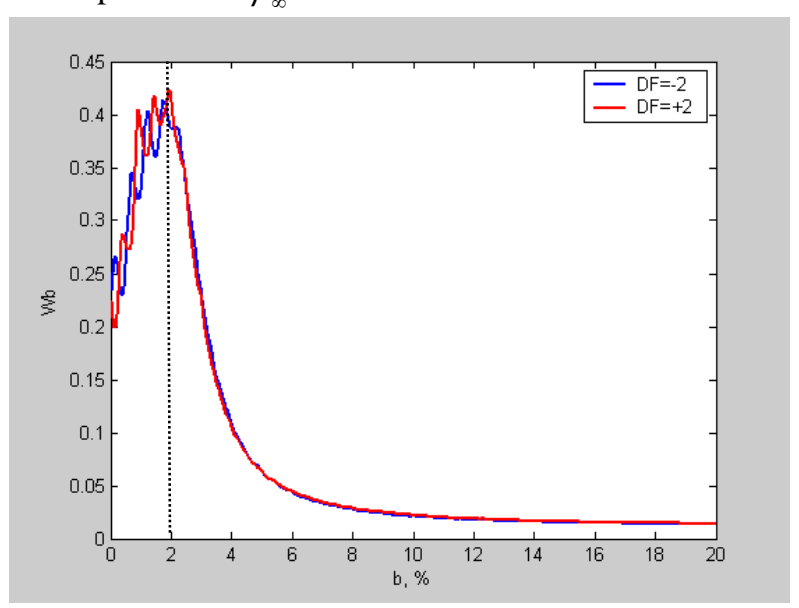

Figure 3. Dependence of the absorption function $W b$ on the dimensionless modulation frequency $b$ at a fixed value of $D F$ and $m=2$. The position of the maximum at $D F=+2$ is marked with a dashed line.

Modulation of radiation at frequencies (1 ... 3)\% of the fundamental frequency increases the absorption by $1,5 \ldots 2$ times. Increasing the modulation frequency further decreases absorption. Thus, there is an optimal 
value of the modulation frequency, which should be automatically determined during the experiment.

We turn to the description of the dynamics of the size of population in the presence of external influence. Fig. 4 shows an S-model for studying population dynamics in accordance with the modified Verhulst equation (6). The "Influence_var" block forms variable parameters in accordance with the formulas (7), the "Dyn_Population" block solves the equation (6). The output signal $N(t)$ is displayed on the virtual oscilloscope and transferred to the external program "M_Verh_var", from which the S-model is started. The program sets the values of the parameters at certain time intervals, if necessary, optimizes the value $\beta_{\infty}$ and builds graphs of the dependence $N(t)$. Simulation time is measured in division time units: $\Delta T=1$, characteristic times $\tau_{\alpha}=100, \tau_{\beta}=3$, the simulation time parameter is 9 , the simulation step is 0,0025 .

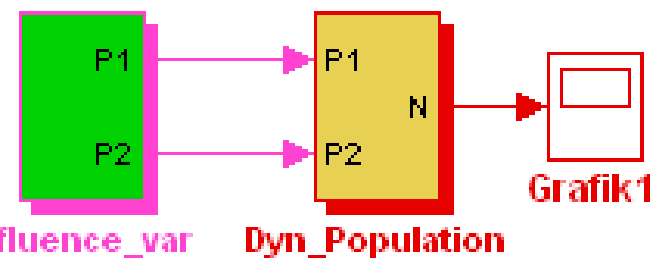

Figure 4. S-model for studying population dynamics In Fig. 5 shows graphs describing the dynamics of the population size for various values of the parameter of the effectiveness of the impact $\beta_{\infty}$. It follows from the graphs that the hormesis time is from 0,5 to 1,5 periods of generation, followed by a section in which the dependence $N(t)$ is close to linear. The linear section has a size up to 2 generation periods. It is on this section that the procedure for optimization (automatic tuning) of the parameters of the modulated radiation is supposed to be carried out. The autotuning interval is indicated in Fig. 5 dotted lines.

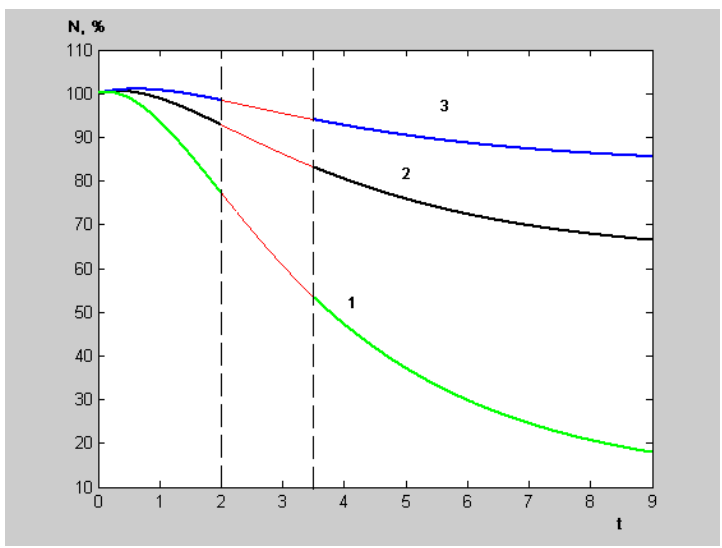

Figure 5. Population dynamics at impact efficiency values $\beta_{\infty} \cdot 1-\beta_{\infty}=0,9 ; 2-\beta_{\infty}=0,5 ; 3-\beta_{\infty}=0,2$.

4 Simulation of a control system with automatic optimization of the modulation frequency

This section presents the results of preliminary modeling, describes the algorithm of operation of the block for automatic tuning of the modulation frequency during the experiment, and shows the operation of a computer model of a control system with an automatic tuning block.

\subsection{Preliminary modeling results. Optimization problem statement}

The dependence of the absorption function $W$ on the parameters specified in (10) was investigated numerically at the values $0 \leq D F \leq 5 \%, \quad 0 \leq d \leq 0,2, \quad 1 \% \leq b \leq 20 \%$. Fig. 6 shows a plot of the surface at $D F=0$. It follows from the graph that when the radiation frequency coincides with the resonant frequency, modulation leads to a decrease in the effectiveness of the impact. This is as it should be, since single-tone modulation, while broadening the signal spectrum, simultaneously reduces its power at the center frequency.

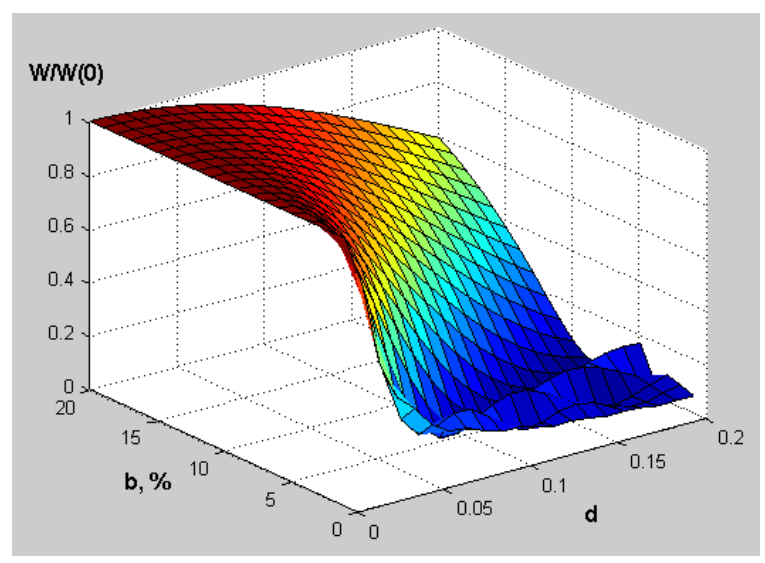

Figure 6. Dependence of the absorption function on the parameters $d$ and $b$ at $D F=0$.

In Fig. 7 shows the surfaces at $D F=2 \%$ and $D F=$ $5 \%$. It follows from the graphs that for a modulated signal there are regions of parameters $d$ and $b$, in which the absorption increases in comparison with unmodulated radiation. 


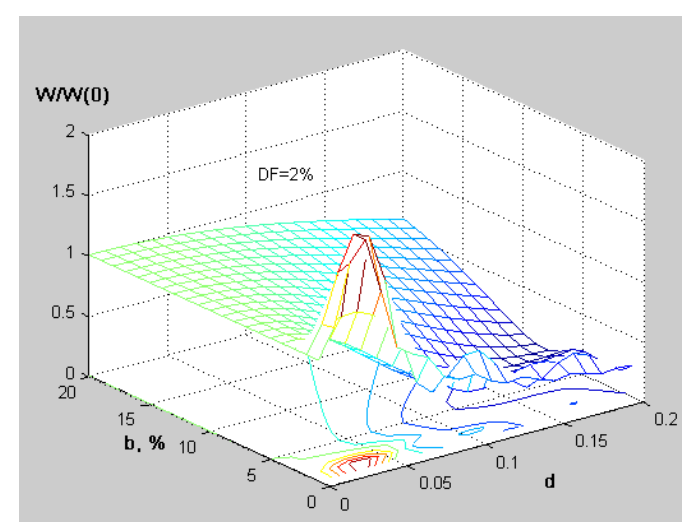

a)

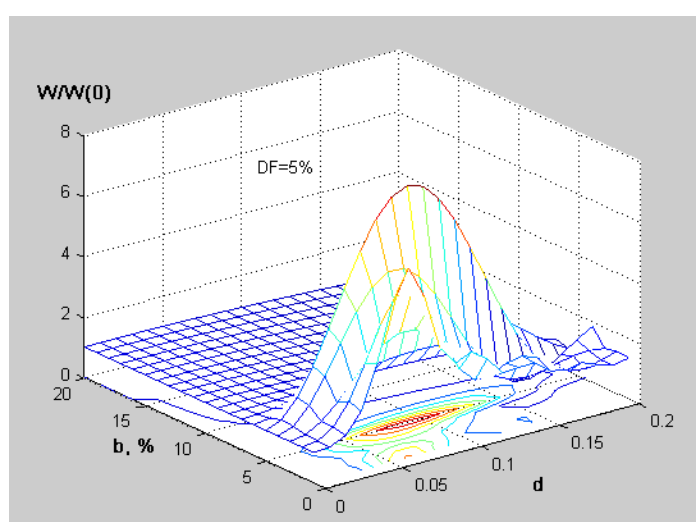

b)

Figure 7. Dependence of the absorption function on the parameters $d$ and $b$ at $D F=2 \%(a)$ and $D F=2 \%(b)$.

At $D F=2 \%$ absorption increases $1,5 \ldots 2$ times, with $D F=5 \%-6 \ldots 8$ times. A qualitative analysis of the research results shows that the regions of extreme values of the function $W$ arise at $d / b \square 1 \ldots 3$. According to definition (11), this means that at the modulation index $m=2$, the absorption function $W b$ always has a maximum, which is not absolute, but close to it.

Thus, the results of preliminary modeling allow us to conclude that under conditions of uncertainty of the resonant frequency, optimization can be carried out in one parameter - the modulation frequency $F m$, with a fixed value of the modulation index $m=2$.

\subsection{Algorithm for determining the optimal modulation frequency in the experiment.}

It is proposed to carry out automatic tuning in a system with feedback, where the parameter that determines the effectiveness of the control action is the modulation frequency of microwave radiation $\mathrm{Fm}$, and the measured output is the population size $N(t)$.

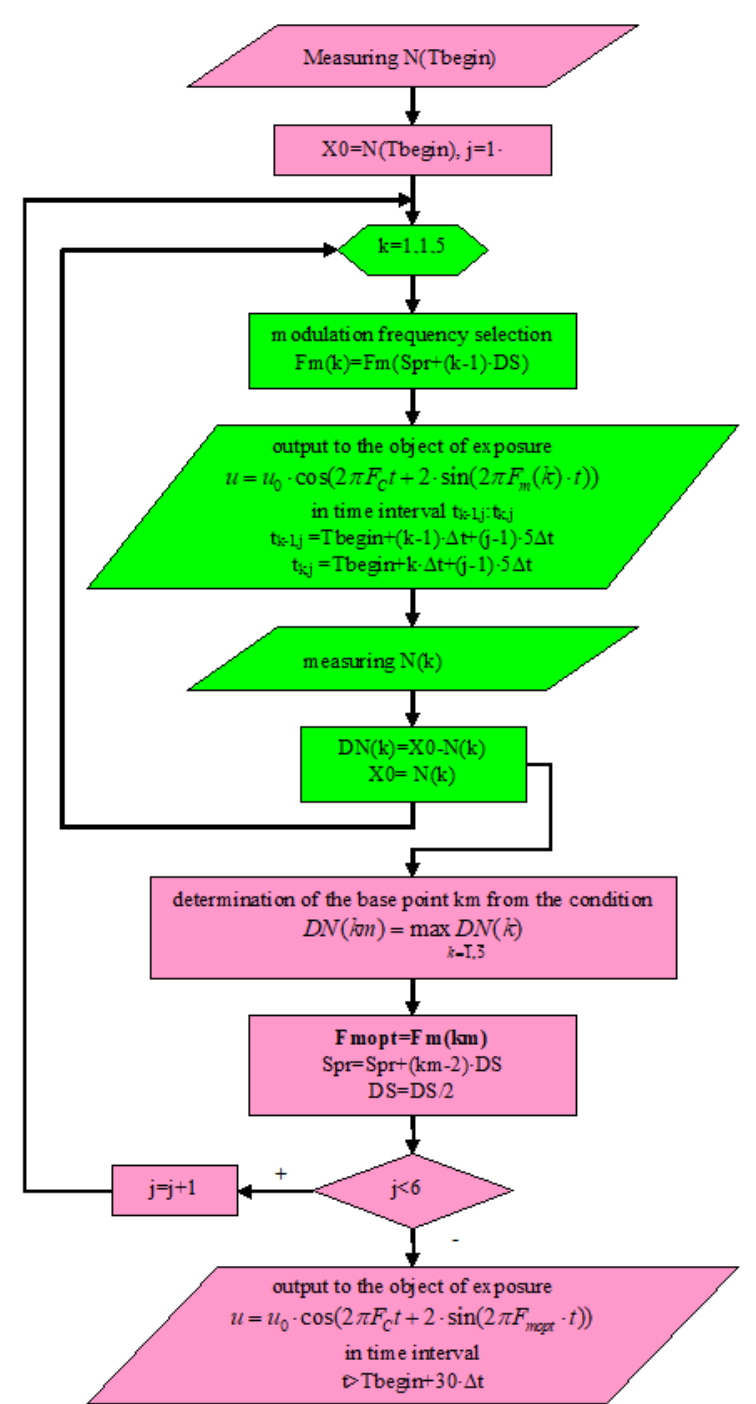

Figure 8. Block diagram of the algorithm for automatic tuning of the modulation frequency

The value of $N(t)$ can be measured in real time using one of the express methods specified in [OFS.1.7.2.0008.15, 2015]: counting under a 
microscope using a counting chamber, conductometric method, etc. Since the effectiveness of the action is determined by the parameter $\beta_{\infty}$, which, in accordance with equation (2.6), determines the rate of decrease in the number in a linear section, the goal of optimal control (2.9) can be reformulated as follows:

$$
\Delta N_{k}=N\left(t_{k-1}\right)-N\left(t_{k}\right) \rightarrow \max , \quad t_{k}-t_{k-1}=\Delta t .
$$

The sampling interval is chosen so that it is possible to measure the change in the population size during this time, i.e. the interval should not be very small. On the other hand, the tuning must be completed within the time indicated in Fig. 5, which imposes restrictions on the number of optimization steps. Recall that tuning begins after the end of the hormesis process.

There are many numerical methods for solving extreme problems of unconstrained optimization: zero-order methods, gradient methods, second-order methods [Ivanov, 2006]. Since in our case the number of optimization steps is limited, it is proposed to apply the simplest method of direct zero-order search to solve the problem. The search is carried out in the range of modulation frequency values from 0 (no modulation) to $\delta F_{\mathrm{m}}$. The initial search step is selected: $D S=\delta F_{\mathrm{m}} / 4$, measurements are taken with the specified step for 5 equally spaced values of the modulation frequencies. The value at which condition (4.1) is satisfied is selected as the basic one, similar to the Hook-Jeeves configuration method, for the next iteration. Then the search is repeated around the base point with a step in modulation frequency halved. The number of iterations is determined by time constraints.

After completing the adjustment, the action is carried out with the found value of the modulation frequency, the measurements are stopped (open control system [Oshchepkov, 2013]). The block diagram of the described algorithm is shown in Fig. 8 for six iterations.

\subsection{Modelling of the control system operation with automatic optimization of the modulation frequency}

To modelling the operation of the control system with automatic optimization of the modulation frequency, the programs "Modul_f" and "M_Verh_var" were used. The "Modul_f" program was used to determine the dimensionless absorption function $W b$ at $m=2$ and a given value of the $D F$, which simulates the response of an object to an external impact. To implement the search algorithm shown in Fig. 8, the function was represented by a $1 \times 513$ array in the range $b=0 \ldots 8 \%$. The program "M_Verh_var" started automatic tuning of the $b$ parameter at the moment Tbegin $=2$ with a sampling step $\Delta t=0,05$. The tuning was carried out in 30 steps and took 1,5 generation periods. The program determined the optimal $b_{\text {opt }}$ value of the $b$ value, at which the maximum of the $W b$ function is reached, and after the end of the tuning it supplied this value to the object.

An example of the control system operation with an automatic tuner is shown in Fig. 9 for $D F=2 \%$. Exposure without frequency modulation in this case reduces the initial number taken as a unit to 0.78 ; exposure with an optimized modulation frequency leaves the number at the level of 0.58 , i.e. the effectiveness of the impact increases by 1.3 times.

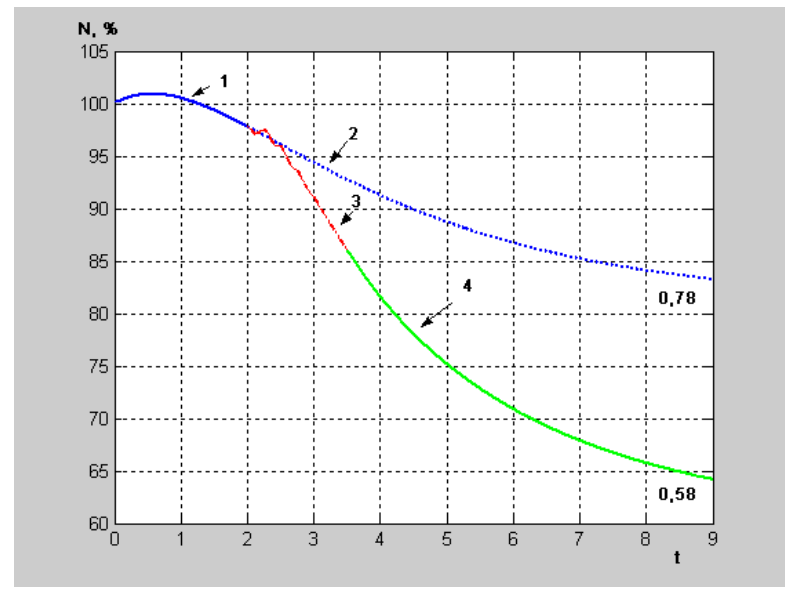

Figure 9. An example of the operation of a control system with an automatic tuning block for $D F=2 \%$ 1 - the interval of hormesis; 2 - population dynamics under the influence of unmodulated radiation; 3 - the interval of work the automatic tuning block; 4 population dynamics under the influence of radiation with optimized frequancy of modulation.

The limit values of the population size are indicated.

The results of the program for the uncertainties of the resonant frequency in the range from $-5 \%$ to $+5 \%$ are presented in the Table. The results show that in the case of an exact coincidence of the radiation frequency with the resonant frequency, the algorithm automatically enters the operation mode without modulation $\left(b_{\text {opt }}=0\right)$, with DF uncertainties from 1 to $5 \%$, the algorithm retains the effect efficiency at the level of $35 \ldots 65 \%$. 
Table 1. Optimal values of the modulation frequency

\begin{tabular}{c|c|c|c}
\hline$D F, \%$ & $W b(0)$ & $b_{\text {opt }}, \%$ & $W b\left(b_{\text {opt }}\right)$ \\
\hline \hline-5 & 0.047 & 5.06 & 0.334 \\
-4 & 0.072 & 3.94 & 0.3436 \\
-3 & 0.120 & 2.81 & 0.360 \\
-2 & 0.234 & 1.75 & 0.414 \\
-1 & 0.552 & 0.69 & 0.622 \\
0 & 1 & 0 & 1 \\
+1 & 0.539 & 0.38 & 0.675 \\
+2 & 0.224 & 1.94 & 0.424 \\
+3 & 0.113 & 2.88 & 0.376 \\
+4 & 0.066 & 3.81 & 0.358 \\
+5 & 0.043 & 4.69 & 0.346 \\
\hline
\end{tabular}

\section{Conclusion}

Frequency modulation of microwave radiation under conditions of uncertainty of the resonant frequency allows you to provide an acceptable level of the inhibitory effect of the electromagnetic field on microorganisms, provided that the modulation frequency is selected optimally. A fast and effective way to optimize is to automatically tuning the modulation frequency using the search algorithm described in this paper.

\section{References}

Baskakov, S.I. (2016). Radio circuits and signals. URSS. M. (In Russian).
Fursova, P.V., Terlova, L.D., Riznichenko, G. Yu. (2008). Mathematical models in biology. RKhD. M-Izhevsk. (In Russian).

Ikhlov, B.L., Oshchepkov, A.Yu., Volkhin, I.L. (2020). About the possibility of application of a non-thermal microwave in medicine. In Journal of New Medical Technologies. 3:99-102. (In Russian).

Ivanov, V.N. (2006). Numerical methods for nonlinear programming. PSU. Perm. (In Russian).

Kovaleva N.A., Manevich L.I., Musienko A.I., Savin A.V. (2009). Low-frequency localized vibrations of the DNA double helix. In High-molecular compounds, ser. A, v. 51, pp. 1174-1188. (In Russian).

Kozmin, G. V., Egorova, V. I. (2006). Stability of biocenoses under conditions of changing electromagnetic properties of the biosphere. In Biomed. Tekhnologii i radioelektronika. V. 3, pp. 61-72. (In Russian).

Kuzin, A.M. (1991) The problem of small doses and the idea of hormesis in radiobiology. In Radiobiologiya, vol. 31, is.1, pp. 16-21. (InRussian).

Landau, L.D., Lifshiz, E.M. (1988). Mechanics. Nauka. M. (InRussian).

OFS.1.7.2.0008.15. (2015) Determination of the concentration of microbial cells. State Pharmacopoeia of the Russian Federation. XIII edition. Volume II. (In Russian).

Oshchepkov, A.Yu. (2013). Automatic control systems: theory, application, simulation in MATLAB. Lan. SPb. (In Russian)

Oshchepkov, A.Yu. (2018). Computer model for research the dynamics of populations of microorganisms in the presence of external control influences. In Vest. Perm. un-ta, sir. Information systems and technologies. pp. 40-46. (In Russian). 